\title{
- Global inventory of landscape patterns and latent variables of landscape spatial configuration
}

\author{
Jakub Nowosad, Tomasz F. Stepinski* \\ Space Informatics Lab, Department of Geography and GIS, University of Cincinnati, Cincinnati, USA, OH 45221-0131, USA
}

\begin{abstract}
We present a regionalization of the entire Earth's landmass into land units of homogeneous landscape patterns. The input to the regionalization is a high resolution Global Land Cover (GLC) dataset. The GLC is first divided into local landscapes - small non-overlapping square blocks of GLC cells. These blocks are agglomerated into much larger land units using a pattern-based segmentation algorithm. These units are tracts encompassing cohesive patterns of land cover and the procedure divides the entire landmass into tracts of land with discernibly different patterns. We characterize a pattern in each unit by a set of 39 landscape metrics. The resulting spatial database of land units is the major product of this study. We make this database freely available to the community in order to provide foundational information for studies aiming at explaining relationships between landscape pattern and ecological process and between the process and patterns and their controlling factors. The procedure of obtaining the database is described, the quality assessment of units delineation is given, and the statistics of the major properties of the units are presented. To showcase the utility of the new database we use it to demonstrate that a variability of geometric configurations of landscape patterns worldwide can be captured in terms of only two variables - complexity and aggregation - as they explain $70 \%$ of the variability. This allows for a meaningful, two-dimensional classification and mapping of landscape patterns on the basis of their geometry. Such mapping reveals that the majority of terrestrial landscapes are characterized by a simple, frequently monothematic, pattern of land cover. Thus, landscapes on Earth are mostly segregated by the land cover type and complex landscapes with a diverse mix of different land cover types are rare exceptions from the prevailing monothematic cover.
\end{abstract}

Keywords:

Global land cover, spatial pattern, regionalization, landscape metrics, complexity, aggregation

\section{Introduction}

Global land cover (GLC) maps are obtained by classifying pixels in a global mosaic of Earth observation (EO) images into several categories of Earth's surface properties. The spatial resolution of GLC maps ranges from $30 \mathrm{~m}$ to $1 \mathrm{~km}$ (Chen et al., 2014; Pfeifer et al., 2012; Tuanmu and Jetz, 2014; Tsendbazar et al., 2015) while their thematic resolution (number of categories) ranges from 10 to 27. The importance of land cover maps for global ecology stems from the fact that they could be used to provide the first-order information about geographical distribution of biodiversity and ecological processes (Siriwardena et al., 2000; Eyre et al., 2004; Heikkinen et al., 2004; Fuller et al., 2005; Luoto et al., 2006)

However, frequently it is a spatial pattern of land cover categories rather than a category itself that is of environmental or ecological interest. This is because grid cells of GLC maps are too small units of an area to be used for analysis on regional, continental or global scale. At such coarse scales a landscape pattern (LP) - an area having discernibly cohesive spatial arrangement (mosaic) of land cover categories - is a more natural unit of analysis (Wickham and Norton, 1994; Riitters et al., 2000; Riitters, 2011; Omernik and Griffith, 2014). A regionalization of GLC into LPs

${ }^{*}$ Corresponding author

Email addresses: nowosad.jakub@gmail.com (Jakub Nowosad), stepintz@uc .edu (Tomasz F. Stepinski) 
would delineate naturally occurring land units which are likely to be environmentally homogeneous and may serve as the first order approximation to global ecoregions (Bailey, 1989; Olson et al., 2001).

Regionalization of a land cover map into LPs was first proposed by Wickham and Norton (1994). However, because at that time the process of pattern-based regionalization could only be performed manually, the concept was not widely used until algorithmic methods of regionalization become available. Pattern-based units are sometimes easy to see on a land cover map but they are always difficult to delineate by manual means (especially over large spatial extent) as no two analysts are likely to arrive at the same partitioning. Only algorithmic regionalization can assure reproductivity of partitioning and it is the only practical means for partitioning large (continental, global) land cover maps.

At first, algorithmic regionalization of land cover maps was applied only to delineate different forest patterns (Long et al., 2010; Kupfer et al., 2012), but later it was also applied to delineate multi-categorical patterns (Niesterowicz and Stepinski, 2013; Partington and Cardille, 2013; Niesterowicz et al., 2016). All regionalization algorithms delineate LPs by agglomerating local landscapes - a small block of land cover cells whose categories form a pattern on the scale defined by the size of the block. Approaches to algorithmic regionalization differ by how local landscapes are described, compared, and agglomerated into LPs (Niesterowicz and Stepinski, 2016). Algorithmic regionalization of GLC had not been previously attempted due to computational challenges associated with the large size of a GLC dataset.

This paper has two major contributions. (1) We algorithmically regionalize a global GLC and obtain a SQLsearchable GIS database containing the global inventory of land units of cohesive land cover patterns. Global regionalization of GLC is made possible by utilizing a segmentation technique instead of clustering technique to agglomerate local landscapes. Segmentation technique also allows the results to be in the form of the GIS database with each LP described by a list of attributes that include landscape metrics (Haines-Young and Chopping, 1996). We make the database available to the community in hope that it can support a range of investigations pertaining to environmental conservation, planning, and ecology. (2) Using the newly created database, we demonstrated that the variance in spatial (geometric) configurations of landscape mosaics worldwide is sufficiently captured (71\%) by only two variables which we call complexity and aggregation. This finding facilitates classification of LPs with respect to their geometry - the first step to a complete classification of land cover patterns.

\section{Methods}

In this section, we describe a GLC dataset we used as an input, a principle and a technique of our pattern-based regionalization method, our selection of landscape metrics, and PCA-based analysis of metrics variability.

\subsection{Data}

We use the CCI-LC 2010 dataset (http://maps.elie.ucl.ac.be/CCI/viewer/) as an input for regionalization of LPs. The CCI-LC dataset is a product of ESA Climate Change Initiative (CCI) to produce a temporal series of GLC maps which are as accurate and multi-year compatible as possible so they can be used in climate modeling. CCI-LC maps are available for several epochs; we use the 2010 map. The CCI-LC map is in the form of $64,800 \times 129,600 \mathrm{Lat} / \mathrm{Lon}$ grid, thus its spatial resolution is 10 arc-sec or $\sim 300 \mathrm{~m}$ at the equator. Each grid cell is classified into one of 22 categories (see Fig. 2 for a legend) based on the FAO/UNEP Land Cover Classification System (LCCS).

\subsection{Regionalization of landscape patterns}

Regionalization of LPs is performed using the Geospatial Pattern Analysis Toolbox (GeoPAT) (Jasiewicz et al., 2015, 2017) - a collection of GRASS GIS (GRASS Development Team, 2016) modules for carrying out pattern-based analysis of large categorical grids, such as the CCI-LC. The entire CCI-LC grid is first tessellated into small square blocks (of the size $k \times k$ of CCI-LC cells) to form a new, $k^{2}$ coarser, grid of blocks. A mosaic of land cover categories within each block encapsulates a local pattern, and the segmentation of the grid of blocks aggregates adjacent blocks into bigger land units while preserving the cohesion of the pattern.

Segmentation is the process of partitioning a grid (commonly a digital image but in our case a categorical raster map) into multiple segments in a way that maximizes homogeneity (of a pattern in our case) within segments and dissimilarity (of pattern) between adjacent segments (Haralick and Shapiro, 1985). The segmentation algorithm in 


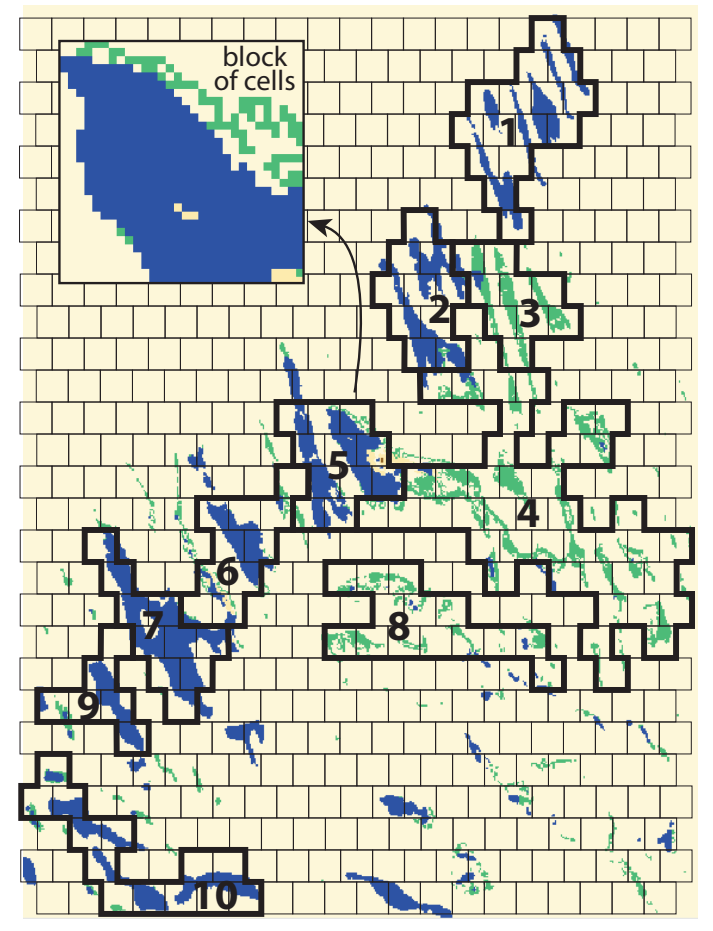

Figure 1: Illustration of pattern-based segmentation using a site located in the Simpson Desert Regional Reserve, Australia. The CCI-LC is tessellated into $9 \mathrm{~km}$-sized $(30 \times 30$ cells $)$ blocks shown by thin lines. The grid of blocks is segmented on the basis of pattern similarity, only 10 regions (thick lines) are shown. The inset shows a sample block in details. Land cover categories present: blue - water, green - wetlands, beige bare lands.

GeoPAT is based on the principle of seeded region growing (SRG) (Adams and Bischof, 1994) but has a number of features that distinguish it from image segmentation algorithms. It segments a grid consisting not of single-category cells but of blocks having complex content (a pattern of different categories) and a non-negligible spatial extent. Because of the non-negligible size of the blocks, the spatial organization of their grid is not rectangular but instead, it consists of alternating horizontal layers of blocks with each layer shifted a half block length with respect to the previous one like in masonry. Such grid is easy to set and it is a sufficiently good approximation of a preferred isotropic hexagonal grid which is difficult to set but, because of its isotropy, minimizes segments' artifacts associated with tessellation.

Fig. 1 illustrates the concept of the grid of blocks and its segmentation. A $252 \mathrm{~km} \times 180 \mathrm{~km}$ fragment of CCILC located in the Simpson Desert Regional Reserve, Australia is shown with the grid of 9 km-sized square blocks superimposed on the map. This site was selected as an illustrative example because it contains only three land cover categories (bare areas, water bodies, and wetlands) that form simple patterns. Ten examples of regions with cohesive LPs are shown as aggregated by the segmentation algorithm.

The pattern within each block is mathematically described by a normalized histogram (the sum of all its bins equals to 1) of land cover category co-occurrence pattern features (Barnsley and Barr, 1996; Chang and Krumm, 1999). Briefly, pattern features are the pairs of land cover categories assigned to two neighboring cells. Histogram counts and bins the features from eight co-occurrence matrices calculated for eight different displacement vectors along the eight principal directions (see Niesterowicz et al. (2016) for an illustrative example). The result is a histogram with $\left(M^{2}+M\right) / 2$ bins, where $M$ is the number of land cover categories; for CCI-LC the histogram has 253 bins. Such histogram describes (indirectly but effectively, see Niesterowicz and Stepinski (2016)) both, composition as well as the spatial configuration of land cover categories within a block and thus the pattern of land cover within a block.

Segmentation algorithm must be able to measure a value of dissimilarity between the blocks. We use the JensenShannon Divergence (JSD) (Lin, 1991) as a measure of dissimilarity between two blocks represented by corresponding 
Table 1: Landscape metrics used to characterize regions

\begin{tabular}{l|l}
\hline Type & Landscape-level metrics \\
\hline \hline \multirow{3}{*}{ Shape } & PAFRAG-Parameter-area fractal dimension; CONTIG_AM - Contiguity index area-weighted average; \\
& CONTIG_RA - Contiguity index range \\
Aggregation & AI - Aggregation index; CONTAG - Contagion; IJI - Interspersion \& Juxtaposition index; PLATJ \\
& - Percentage of like adjacencies, PD - Patch density; DIVISION - Landscape division index; LPI - \\
& largest patch index \\
& COHESION - Patch cohesion index \\
Connectivity & SHDI - Shannon's diversity index; SIDI - Simpson's diversity index; MSIDI - Modified Simpson's's \\
Diversity & diversity index; SHEI-Shannon's evenness index; SIEI - Simpson's evenness index; MSIEI- Modified \\
& Simpson's evenness index \\
\hline
\end{tabular}

normalized histograms $M_{1}$ and $M_{2}$. The JSD expresses the informational distance between the two histograms as a deviation between the Shannon's entropy of the conjugate of the two histograms $\left(M_{1}+M_{2}\right) / 2$ and the mean entropy of individual histograms $M_{1}$ and $M_{2}$. The value of JSD, denoted by $d\left(M_{1}, M_{2}\right)$, is given by the following formula:

$$
d\left(M_{1}, M_{2}\right)=H\left(\frac{M_{1}+M_{2}}{2}\right)-\frac{H\left(M_{1}\right)+H\left(M_{2}\right)}{2}
$$

where $H(M)$ indicates a value of the Shannon's entropy of the histogram $M$ :

$$
H(M)=-\sum_{i=1}^{|M|} m_{i} \log _{2} m_{i}
$$

where $m_{i}$ is the value of $\mathrm{i}$ th bin in the histogram $M$ and $|M|$ is the number of bins (the same for both histograms). For normalized histograms the JSD dissimilarity always takes values from 0 to 1 with the value of 0 indicating that two blocks are identical, and the value of 1 indicating maximum dissimilarity (none of the categories existing in one block can be found in the other).

The most important parameter of the segmentation algorithm is the size of the block, $k$, which sets the scale of the pattern. Other parameters are set to default values; see Jasiewicz et al. (2017) for the list of all parameters and their default values.

\subsection{Landscape metrics}

After land units of cohesive LPs are delineated we calculate a set of landscape metrics for each unit to serve as its attributes. Landscape metrics are algorithms that quantify the specific spatial characteristics of an LP. A large number of different metrics have been developed and collected in a single computer program FRAGSTATS (McGarigal et al., 2002). We use FRAGSTATS definitions to calculate a set of 39 landscape-level metrics, 22 of them are percentages of area covered by contributing land cover categories (PLANDs in the landscape metrics nomenclature) - they characterize the composition of the patterns, and 17 of them are configurational landscape metrics (see Table 1 for details). For this calculations, we use our own code optimized for working with 100,000s of landscape units of different sizes and shapes resulting from the regionalization process. The selection of configurational metrics stems from the fact that land units have very different sizes and shapes so we don't use area metrics and we use only those shape metrics that would not be dominated by the shape of the region itself. We also don't use other metrics which returns values in units of area.

\subsection{Principal Components Analysis (PCA)}

A procedure of finding latent variables for characterization of a geometric configuration of LPs relies on the Principal Component Analysis (PCA). Let's denote by $N$ the number of land units resulting from our regionalization. Each unit is described by values of 17 configurational metrics listed in Table 1 (we don't use PLANDs metrics for this analysis). Many of these metrics are correlated and, as a group, they don't represent the major descriptors (latent variables) of pattern configuration. We perform PCA analysis on the correlation matrix (using $N$ values of each metric) to uncover latent variables and thus to reduce the number of variables needed to capture the variability of pattern configurations in our database. Because of this goal, we perform varimax rotation (Kaiser, 1958) on obtained 
principal components. Rotated components are not "principal" inasmuch as they are not uncorrelated, but they can be easier to interpret in terms of original metrics and thus are more useful as sought after latent variables or dimensions of landscape spatial configuration.

\section{Regionalization results}

Using a pattern-based segmentation methodology described in Section 2.2 we regionalized the entire Earth's landmass into regions (segments) containing cohesive land cover patterns. Four regionalizations were calculated distinguishable by the assumed scale of local landscape (size of the block); $30 \mathrm{~km}, 15 \mathrm{~km}, 9 \mathrm{~km}$, and $6 \mathrm{~km}$. Table 2 summarizes the number of blocks and segments in each regionalization. Increasingly smaller blocks enclose increasingly specific patterns. More specific patterns extend over smaller regions, thus using a smaller block's size leads to a larger number of regions (fourth column in Table 2).

Table 2: Summery of pattern-based regionalizations of CCI-LC using different scales of local landscape

\begin{tabular}{rrrrrr}
\hline scale & block size & \# blocks & \# regions & $\langle$ inhomogeneity $\rangle$ & $\langle$ isolation $\rangle$ \\
\hline \hline $30 \mathrm{~km}$ & $100 \times 100$ & $3,359,232$ & 9,946 & 0.13 & 0.29 \\
$15 \mathrm{~km}$ & $50 \times 50$ & $13,436,928$ & 36,284 & 0.13 & 0.29 \\
$9 \mathrm{~km}$ & $30 \times 30$ & $37,324,800$ & 101,274 & 0.12 & 0.28 \\
$6 \mathrm{~km}$ & $20 \times 20$ & $69,984,000$ & 238,032 & 0.12 & 0.28 \\
\hline
\end{tabular}

The purpose of generating landscape regionalization/landscape database using different block sizes is that they provide worldwide inventories of landscapes at different levels of spatial details. Of the four regionalizations we generated, the one based on $30 \mathrm{~km}$ blocks is the least spatially detailed. On the other hand, it has the smallest number of regions and may be well-suited for applications on the broadest scale. The regionalization based on $6 \mathrm{~km}$ blocks is the most spatially detailed and may be the most appropriate for more spatially focused applications, but it requires an ability to handle a large spatial database. All four regionalizations are available for download from the University of Cincinnati Space Informatics Lab at http://sil.uc.edu. They are in the form of shapefiles with attribute tables containing information about regions location, their area, values of PLANDs, values of 17 landscape metrics listed in Table 1, standardized values of the first 10 principal components, standardized values of the first 10 rotated principal components, and the type of landscape spatial configuration (see section 4)).

The last two columns in Table 2 quantify the quality of regions' delineation. According to Haralick and Shapiro (1985) a segmentation is "good" if patterns within regions are cohesive and when adjacent regions are dissimilar from the focus region. We measure a cohesiveness of regions' patterns using an inhomogeneity metric. Inhomogeneity is a property of a single region; it measures a degree of mutual dissimilarity between all local patterns (blocks) within the region. As a measure of region inhomogeneity we use an average distance between all distinct pairs of blocks in a region. For a region $S$ consisting of blocks $\left(M_{1}, \ldots, M_{k 1}\right)$ the inhomogeneity $\delta$ is given as:

$$
\delta(S)=\frac{1}{k 1(k 1-1)} \sum_{i} \sum_{j \neq i} d\left(M_{i}, M_{j}\right)
$$

as there are $k 1(k 1-1)$ distinct pairs of blocks in the region $S$. The function $d\left(M_{i}, M_{j}\right)$ is given by eq.(1). Inhomogeneity has a range between 0 and 1 , smaller values indicate regions with more cohesive patterns. Average values of inhomogeneity (calculated over all regions in the regionalization) are given in the fifth column of Table 2. Their low values indicate that, on average, regions in all regionalizations are indeed characterized by cohesive patterns of land cover.

We measure a degree to which a region stands out from its neighboring units using an isolation metric. Isolation is also a property of a single unit; it is calculated as the average linkage $\left\langle D\left(S_{0}, S_{\alpha}\right)\right\rangle$ between the focus region $S_{0}$ and all of its immediate neighbors $S_{1}, \ldots, S_{n}$, where $n$ is the number of neighbors and the symbol \langle\rangle indicates averaging. 


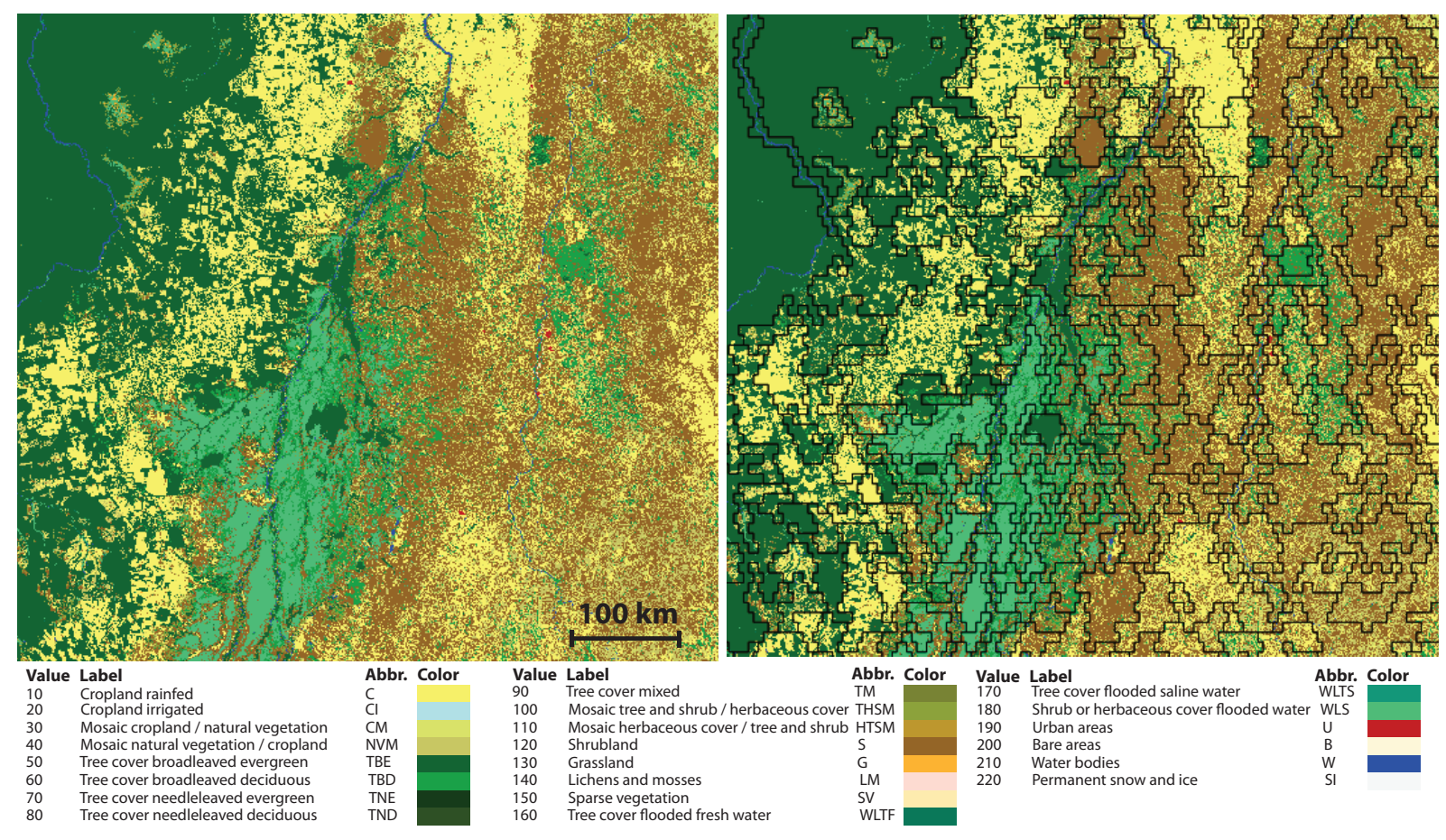

Figure 2: Regionalization of the CCI-LC dataset (using local landscapes at $9 \mathrm{~km}$ scale) shown within the bounds of the site located in the Brazilian state of Tocantins. (Left) CCI-LC map; although some pattern can be seen it would be very difficult to manually delineate them with acceptable precision. (Right) Calculated boundaries of land units overlaid on the CCI-LC map; each unit contains a unique landscape - a cohesive pattern of land cover categories. (Bottom) The CCI-LC legend with category names, codes, and abbreviations.

The linkage (the distance between two groups of blocks in two regions) is given by

$$
D\left(S_{0}, S_{\alpha}\right)=\frac{1}{k_{0} k_{\alpha}} \sum_{i=1}^{k_{0}} \sum_{j=1}^{k_{\alpha}} d\left(M_{0, i}, M_{\alpha, j}\right)
$$

where $k_{0}$ and $k_{\alpha}$ are the numbers of blocks in the focus region and in one of its neighbors, respectively. Isolation has a range between 0 and 1, regions with larger values of isolation stand out more from their neighboring segments. Average values of isolation (calculated over all regions in the regionalization) are given in the sixth column of Table 2. Their values are much higher than the values of inhomogeneity indicating that indeed regions boundaries separate discernibly different patterns.

Fig. 2 shows a fragment of worldwide $9 \mathrm{~km}$-scale regionalization. The site shown is located in the Brazilian state of Tocantins and is centered at $10^{\circ} 37^{\prime} \mathrm{S}$ and $49^{\circ} 05^{\prime} \mathrm{W}$. Because it is located in the central zone of Brazil, the site has characteristics of the Amazon Basin and semi-open pastures. It also includes wetlands around the river Araguaia in the proposed South Amazon Ecotones Ecological Corridor. The left panel of Fig. 2 shows the CCI-LC map over the site, and the right panel shows the same map overlaid by boundaries of calculated regions. It can be visually confirmed that each segment encloses a cohesive pattern of land cover categories. Note that the boundaries have a wiggly appearance due to the size of the tiles ( $9 \mathrm{~km}$ in this example) being much larger than the size of CCI-LC cells.

The sizes (and shapes) of regions vary greatly because spatial extents of various landscapes vary. This can be observed to a limited degree in Fig. 2 where Amazon Tree Cover Broadleaved Deciduous (TBD) region is larger than regions with more complex landscapes. Fig. 3 shows cumulative shares of Earth's landmass occupied by regions of different sizes. This is a log-linear plot constructed for regions delineated using $9 \mathrm{~km}$ scale; plots based on other scales are similar. The horizontal axis shows the logarithms of regions' areas sorted from the largest (left) to the smallest (right); Antarctica is excluded from the cumulative share. Note that regions' sizes vary by several orders of magnitude. The vertical axis shows the percentage of Earth's landmass occupied by regions equal or larger than a given size. Nine 


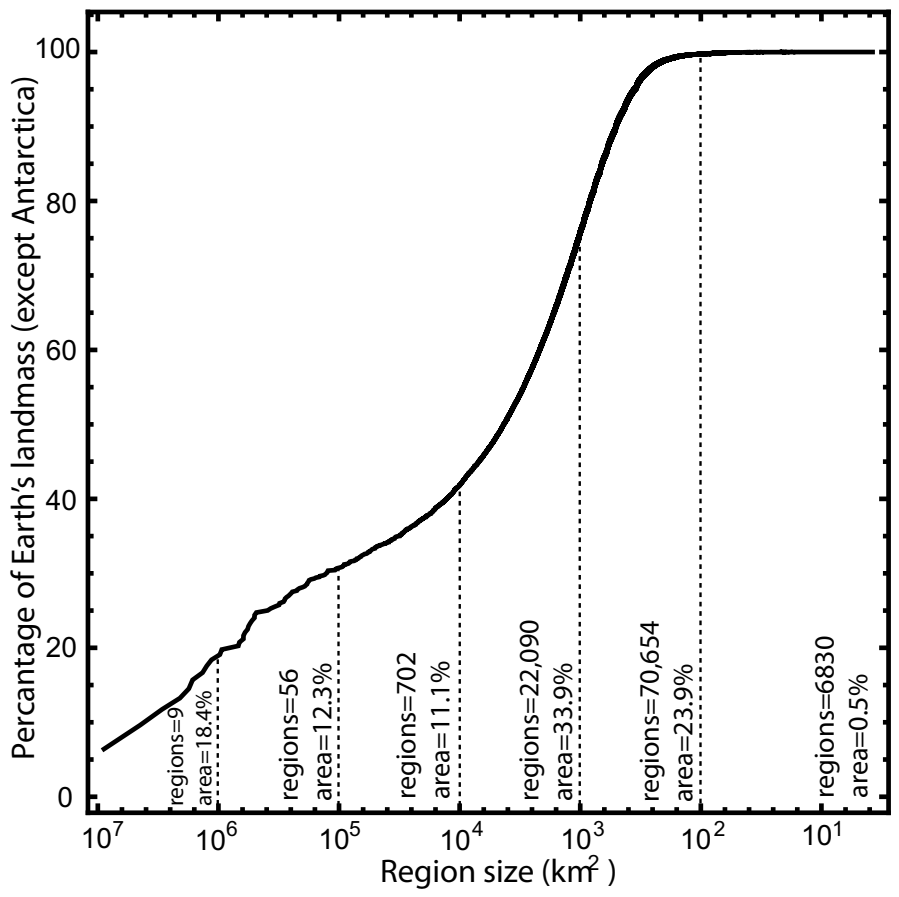

Figure 3: Cumulative share of Earth's landmass (except Antarctica) occupied by land units of different sizes. Units are organized by orders of magnitude of their sizes. Regionalization based on $9 \mathrm{~km}$ scale is used to construct this plot.

regions having areas over 1 million $\mathrm{km}^{2}$ (six desert regions in Africa, Asia, and Australia, ice cover in Greenland, shrub area in North America, and Amazon forest) together occupy over $18 \%$ of the landmass. The largest percentage of the landmass $(\sim 34 \%)$ is occupied by regions having sizes in the range of $1,000 \mathrm{~s} \mathrm{~km}^{2}$.

\section{Finding latent variables of landscape configuration}

In the second part of the paper, we will use the database described in the previous section to find a minimal set of latent variables capable of accounting for a large majority of observed variability in the geometric configuration of LPs. For this, we use the procedure described in section 2.4.

Table 3 lists the percentage of variance explained by the first ten rotated principal components (denoted as (RCs) calculated on the correlation matrix of the set of 17 landscape-level metrics listed in Table 1. The first observation is that the first two RCs explain the lion share of the variance within the set of 17 metrics. Thus, we can neglect remaining components and describe landscape spatial configuration in terms of only two latent variables, $\mathrm{RC} 1$ and $\mathrm{RC} 2$.

The second observation is that percentages of variance explained by top two components depend weakly on the scale of the local landscape. Variance explained by the top two components changes from $71 \%$ for landscapes delineated using a scale of 30 or $15 \mathrm{~km}$ to $58 \%$ for landscapes delineated using a scale of $6 \mathrm{~km}$. In addition, loadings of the two top components remain consistent through various scales, thus their interpretation does not change with the scale. This means that landscapes measured at scales from $6 \mathrm{~km}$ to $30 \mathrm{~km}$ have similar spatial configurations. For further analysis, we use landscapes regionalized assuming the scale of $9 \mathrm{~km}$.

To give an interpretation to the first two RCs we review their loadings - correlations between them and the values of original 17 landscape metrics. Fig. 4 illustrates the loadings of RC1 and RC2. RC1 is positively correlated with all diversity metrics. Diversity metrics are measures of the character of the histogram of a unit's cells with respect to categories of land cover. The value of RC1 increases when the histogram is flat (the unit has a diverse composition of categories) and it decreases when the histogram is peaked (the unit is dominated by a single category). RC1 is 
Table 3: Percentage of variance explained by the first ten rotated principal components

\begin{tabular}{rrrrrrrrrrr}
\hline scale & RC1 & RC2 & RC3 & RC4 & RC5 & RC6 & RC7 & RC8 & RC9 & RC10 \\
\hline \hline $30 \mathrm{~km}$ & 46 & 25 & 8 & 7 & 6 & 4 & 2 & 1 & 1 & 0 \\
$15 \mathrm{~km}$ & 46 & 25 & 8 & 7 & 6 & 3 & 3 & 1 & 1 & 0 \\
$9 \mathrm{~km}$ & 43 & 26 & 8 & 6 & 6 & 4 & 3 & 3 & 0 & 0 \\
$6 \mathrm{~km}$ & 31 & 27 & 12 & 8 & 7 & 7 & 6 & 2 & 1 & 0 \\
\hline
\end{tabular}

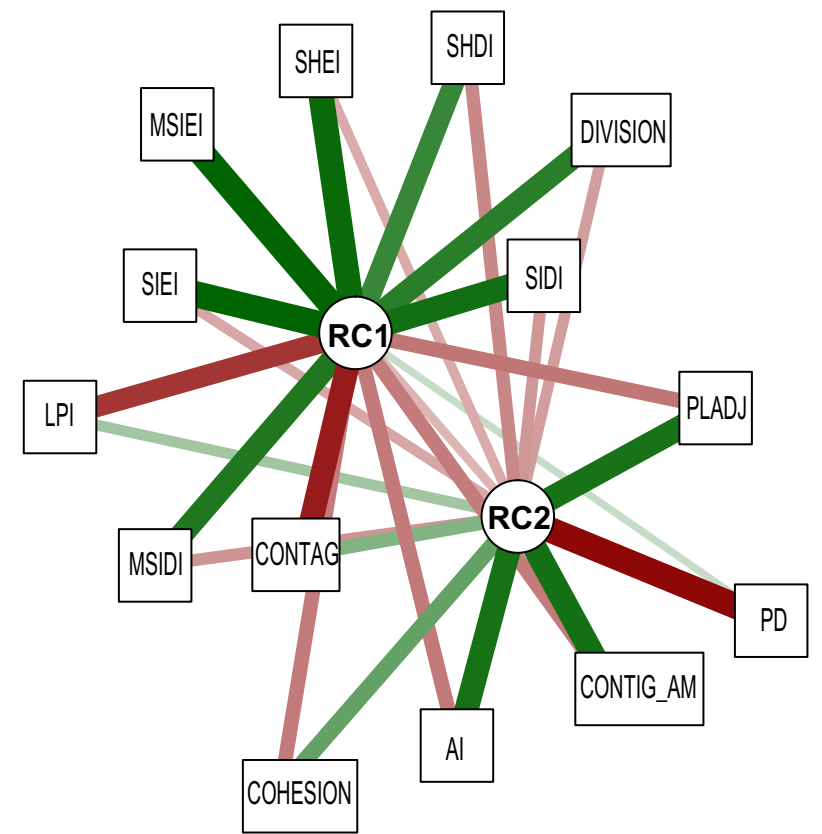

Figure 4: Major loadings of the first two rotated principal components. Positive loadings are indicated by green lines while negative loadings are indicated by red lines; magnitude of a loading is indicated by color intensity and thickness of the line. Three metrics providing negligible loadings are not shown. CONTIG_AM - Contiguity index area-weighted average, AI - Aggregation index, CONTAG - Contagion, PLATJ - Percentage of like adjacencies, $P D$ - Patch density, DIVISION - Landscape division index, LPI - largest patch index, COHESION - Patch cohesion index, SHDI - Shannon's diversity index, SIDI - Simpson's diversity index, MSIDI - Modified Simpson's's diversity index, SHEI - Shannon's evenness index, SIEI - Simpson's evenness index MSIEI - Modified Simpson's evenness index.

negatively correlated with LPI and CONTAG. Thus, the value of RC1 increases if the unit has more category patches and its decreases if the unit has fewer category patches. Overall, we interpret the RC1 as a measure of "complexity" and denote it by the symbol $C$. Small values of $C$ indicate landscape with a simple configuration while large values of $C$ indicate landscape with a complex configuration. RC2 is positively correlated with $A I, P L A D J$, and CONTIG_AM, all measures of aggregation or connectivity. The value of RC2 increases when cells of the same categories are more aggregated within the unit. RC2 is negatively correlated with $P D$, its value increases if a density of patches in the unit decreases. Overall, we interpret RC2 as a measure of "aggregation" and denote it by the symbol A. Small values of $A$ indicate a landscape with a large number of small patches while large values of $A$ indicate a landscape with a small number of larger patches.

\section{1. $C-$ A diagram}

Representing the values of $C$ and $A$ for all units as points $\{C, A\}$ on the two-dimensional plot we obtain a $C-A$ diagram shown in Fig. 5A. To construct this diagram we standardized the sets of $C$ and $A$ values so they have a mean equal to zero and a standard deviation equal to 1 . The $C-A$ diagram shows that the points $\{C, A\}$, which summarize spatial configurations of landscapes worldwide, are not randomly distributed but rather are restricted to a roughly triangular section of a diagram. To get more information from the $C-A$ diagram we impose on it an $8 \times 8$ grid of 

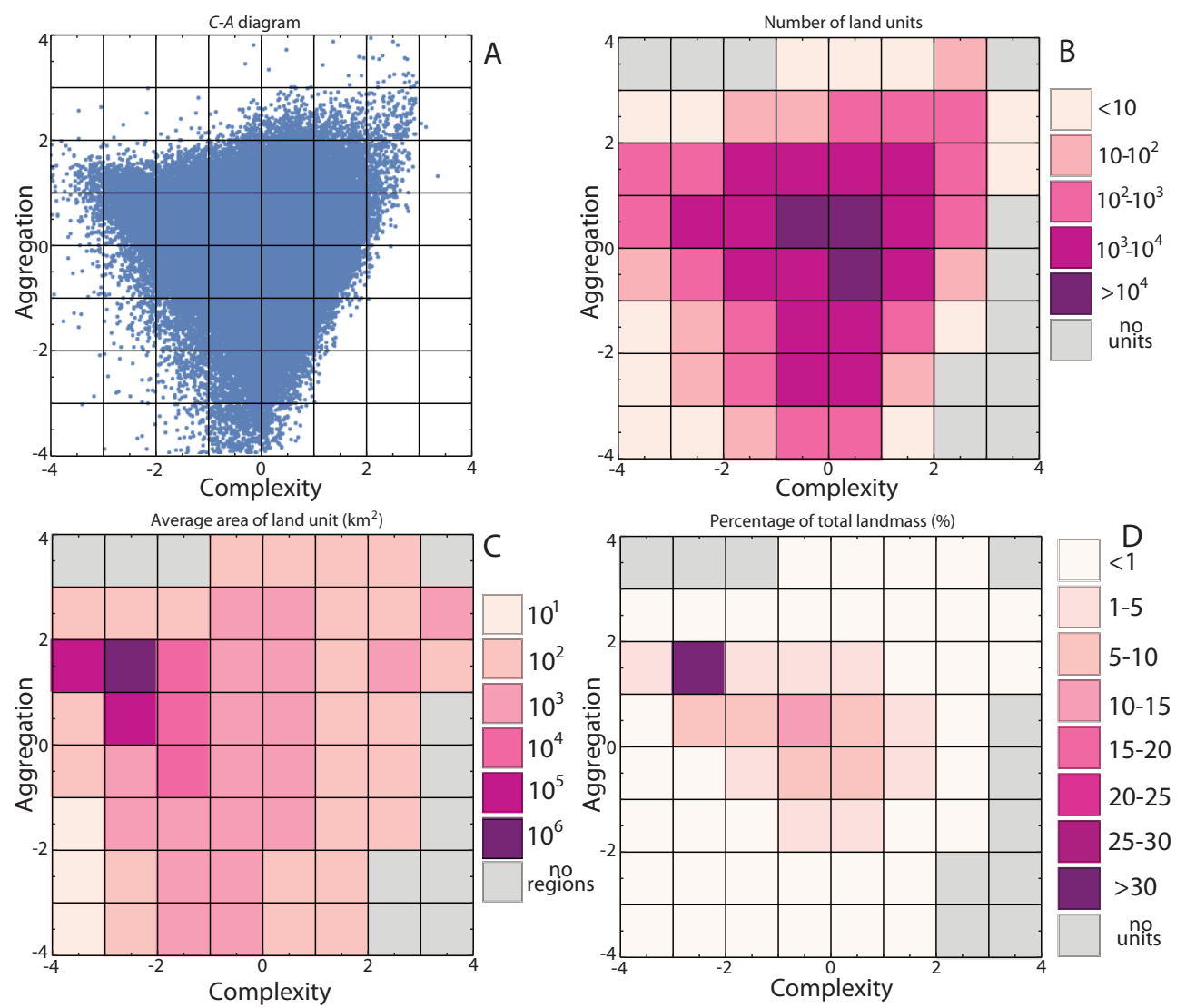

Figure 5: (A) The $C-A$ diagram constructed by plotting points corresponding to values of $C$ and $A$ for all 101,274 land units resulting from regionalization using $9 \mathrm{~km}$ landscape scale. (B) Number of units per sector of the $C-A$ diagram. (C) An area-weighted average area of unit per sector of the $C-A$ diagram. (D) Percentage of the total landmass occupied by all units in a given sector of the $C-A$ diagram.

sectors. Each sector has a size of $1 \times 1$ in dimensionless, standardized units in which $C$ and $A$ are measured. Statistics of land units located in different sectors of the $C-A$ diagram reveals details about an abundance of different pattern configurations.

Fig. 5B shows the number of land units in each sector of the $C-A$ diagram. Note that the entries in the legend increase by an order of magnitude (tens, hundreds, thousands, etc). The largest number of units are in sectors corresponding to the mean values of $C$ and $A$ - most LPs are characterized by medium complexity and aggregation (see middle panel in Fig. 6 for an example of such LP). The number of units in sectors corresponding to values of $C$ and $A$ departing from their mean values decreases steeply but not at the same rate in all directions of the $C-A$ diagram. Fig. 5C shows an area-weighted average area of a unit in different sectors of the $C-A$ diagram. This is to indicate configurations of the largest LPs. By far the largest units are those having simple and aggregated patterns $(-3<C<-2$ and $1<A<2)$. On average, simpler landscapes $(C<0)$ occupy larger areas than more complex landscapes $(C>0)$. Finally, Fig. 5D shows a percentage of total landmass area occupied by all units in a given sector of the $C-A$ diagram. One sector, $-3<C<-2$ and $1<A<2$, consisting of simple and aggregated regions, occupy $31 \%$ of the total landmass. Landscapes characterized by the values of $C$ and $A$ within a standard deviation from their means occupy between 5\%-15\% of landmass per sector, the remaining landscapes occupy small (below 1\%) parts of the landmass.

Fig. 6 shows examples of nine landscapes organized in a grid according to their values of $C$ and $A$. The values of $C$ change horizontally from small (left) to large (right) and the values of $A$ change vertically from small (bottom) to large (top). The top row shows a progression of aggregated landscapes from least complex to most complex. The bottom row shows a progression of disaggregated landscapes from least complex to most complex. 


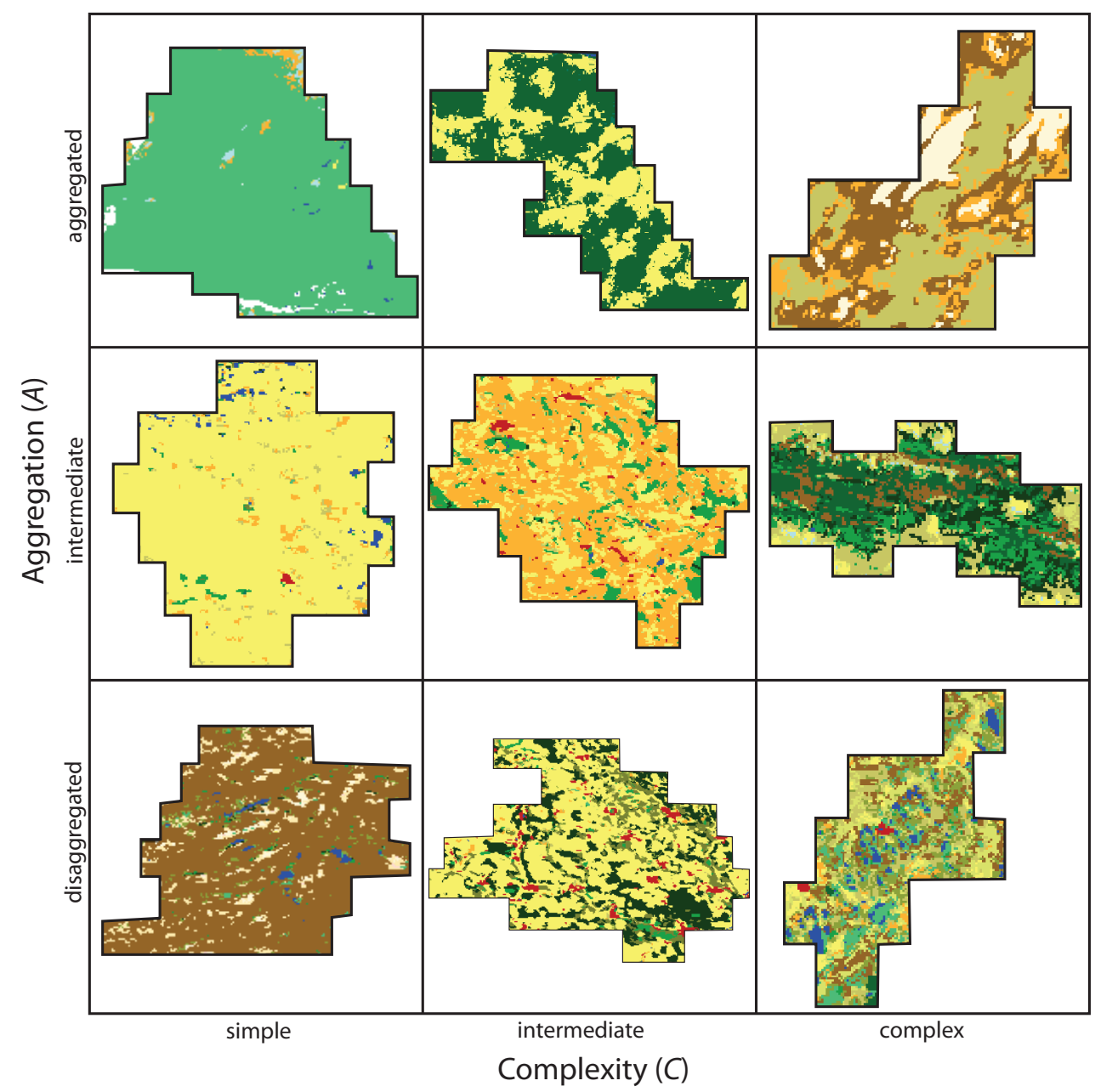

Figure 6: Examples of dependence of landscape spatial configuration on two parameters, complexity $C$ and aggregation A. Spatial scale of each landscape can be identified by the $9 \mathrm{~km}$ size of a single block. For the CCI-LC legend see Figure 2.

\subsection{Mapping landscape configuration types}

Units in our database may be classified into a number of landscape configuration types on the basis of their values of $C$ and $A$. Our database includes a classification obtained by dividing the $C-A$ diagram into nine sectors (instead of 64 sectors as seen in Fig. 5A). All land units for which values of $C$ and $A$ fall within a given sector are given a pattern configuration label corresponding to this sector. Fig. 6 acts as a rough guide to a character of pattern configurations in the nine classes. However, we stress that this classification does not take into account pattern compositions which may be different from those in examples shown in Fig. 6, thus examples should be evaluated only for a geometry of the pattern and not for categories (depicted as colors).

Using this classification we constructed a global map of LPs configurations. Fig. 7 shows a portion of this map restricted to Europe (so the details could be seen). We use a bivariate color scheme for a legend to this map; bluishgray colors indicate simple configurations while reddish-brown colors indicate complex configurations. The land in Europe is dominated by units with LP configurations characterized by intermediate complexity and intermediate aggregation (see middle panel in Fig. 6 for an example of such unit). In Europe, such units are predominantly mosaics of agricultural land and forest, although other compositions within intermediate $C$-intermediate $A$ configurations are also found. Another two landscape configuration types with significant coverage in Europe are simple $C$-intermediate $A$ (light blue color on the map) and simple $C$-aggregated $A$ (gray color on the map). The first of these two types 


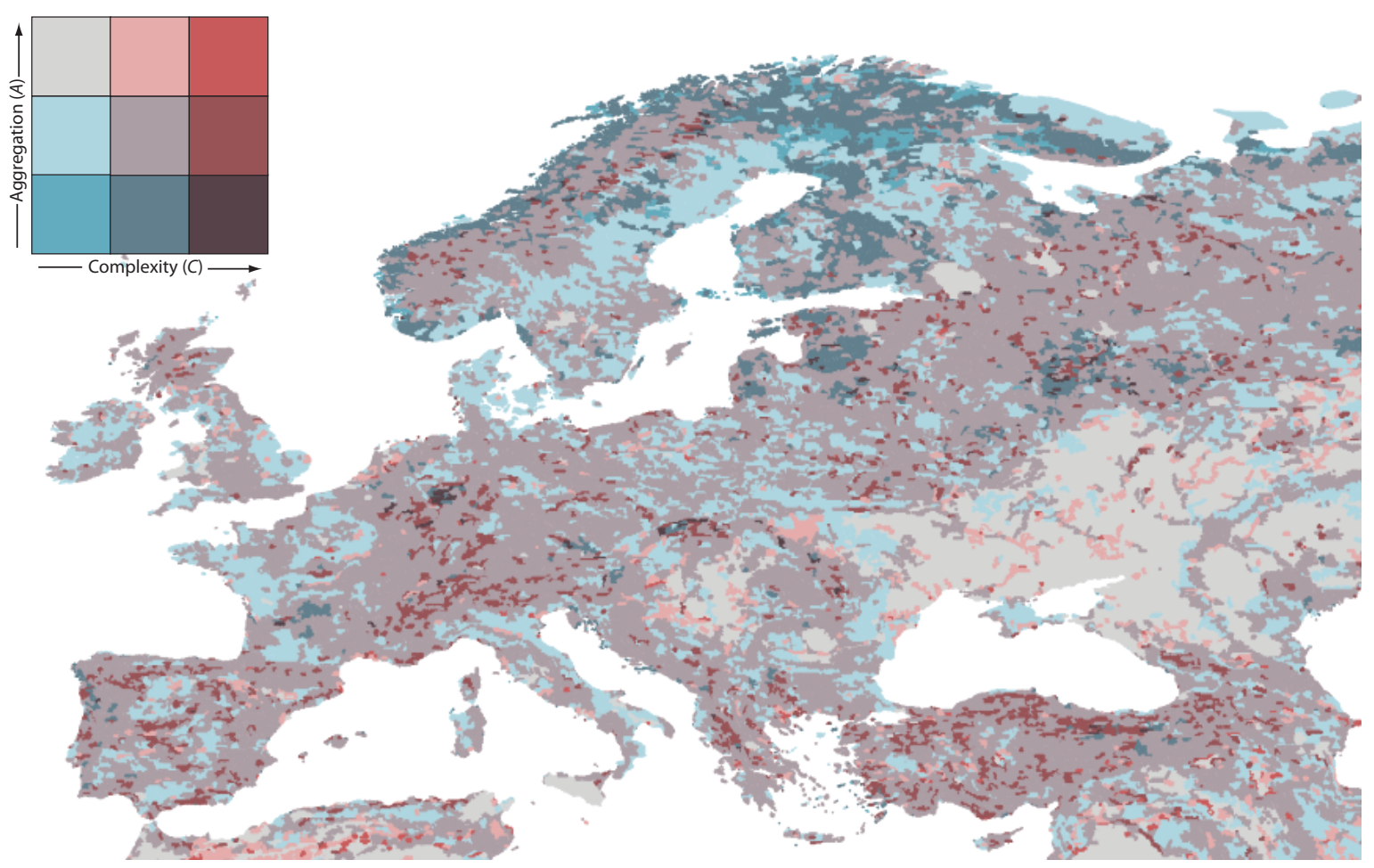

Figure 7: Map of landscape configuration types in Europe using nine categories encapsulating different combinations of complexity (C) and aggregation $(A)$ variables. The legend uses a bivariate color scheme to encode different combinations of complexity and aggregation.

correspond predominantly to agricultural lands with settlements or to needleleaved evergreen forest in Scandinavia, and the second type corresponds to agricultural lands with much fewer settlements (mostly in Ukraine and Russia). Complex landscapes (reddish-brown colors on the map) are rare and occur only in small patches.

\section{Conclusions and discussion}

This paper makes two advances. The first is a development of a worldwide database of LPs with an extensive table of attributes - an important resource for GIS-based studies of global environment and ecology. The second is a demonstration that geometric configurations of LPs around the world can be parametrized by only two latent variables - complexity and aggregation.

A small number of previous studies (Cardille and Lambois, 2009; Partington and Cardille, 2013; Niesterowicz et al., 2016; Niesterowicz and Stepinski, 2017) presented regionalizations based on patterns of land cover, albeit only on a regional scale. In addition, the previous studies (with the exception of Niesterowicz and Stepinski (2017)) were based on clustering rather than segmentation of local landscapes (blocks). Thus, their outputs are classifications of blocks' patterns into LPTs (Wickham and Norton, 1994). An LPT is a category representing all similar LPs. A database which includes only LPTs does not constitute a robust resource to the community because it does not allow for regionalizations using customized criteria. On the other hand, our database contains discernible land units each described by an extensive table of attributes. This allows users to perform custom classifications based on their own criteria. A regionalization of terrestrial landmass into nine types of pattern configuration (section 4.2) is an example of applying one particular set of classification criteria to our database.

Potential applications for our global database of LPs are in the area of broad scale environmental conservation, planning, and ecology. It can be used to explain relationships between landscape pattern and ecological process and between the process and patterns and their controlling factors. Several specific examples of such applications were 
given by Wickham and Norton (1994), they include evaluating the distribution of LPs across ecoregions, assessing a degree of forest segmentation, and evaluating changes of stream density and roads density between different LPs. Other examples include investigating a dependence between LPs and biodiversity (Fahrig, 2003), and evaluating a degree of cohesiveness of climate, pattern of soil types, and patterns of landforms within boundaries of units delineated on the basis of LPs to check for spatial correlation between different physical elements of an environment.

Our second contribution is a demonstration that only two variables, interpreted as complexity and aggregation, are sufficient to explain the variability of geometric configurations of LPs worldwide. This finding follows from the PCA analysis of land cover patterns in the CCI-LC map. To check whether it holds for another depiction of global land cover patterns we have repeated the PCA analysis using the GLC2000 (Bartholome and Belward, 2005). This is the older global land cover map with a legend similar to that of CCI-LC but having the resolution of $1 \mathrm{~km}$ and pertaining to the year 2000. The results of the PCA analysis for GLC2000 are very similar to those obtained for CCI-LC; for 30 $\mathrm{km}$ scale patterns in GLC2000, the top two RCs explain $68 \%$ of the variance (it is $71 \%$ for CCI-LC). The loadings of these top two RCs are also similar to those obtained from the analysis of patterns in the CCI-LC, so their interpretation is the same. We have also repeated the PCA analysis using the NLCD 2011 (Homer et al., 2015). This is the land cover map for the conterminous U.S., its resolution is $30 \mathrm{~m}$, and its 16 land cover categories are different from those in the CCI-LC. The results of the PCA analysis for NLCD 2011 are similar to those obtained for CCI-LC; for the 30 $\mathrm{km}$ scale patterns in the NLCD 2011, the top two RCs explain $61 \%$ of the variance and their loadings indicate that they can be interpreted as aggregation and complexity. This indicates that our findings are robust and they are only weakly dependent on a particular dataset. Their dependence on the assumed scale of local landscapes is also weak (see Table 3).

Our findings can be compared to the previous work of Cushman et al. (2008) on parsimony in landscape metrics. Our analysis differs from that of Cushman et al. in the following way. (A) Utilizing our new database we consider up to 240,000 LPs together covering the entire terrestrial landmass, whereas they considered 528 LPs taken from three sites in the United States. (B) We use as an input a single land cover dataset with a resolution of $300 \mathrm{~m}$, while they used as input three different land cover datasets with resolutions ranging from 25 to 30 meters. (C) Our patternbearing land units vary in sizes and shapes whereas their units are all $7.68 \mathrm{~km} \times 7.68 \mathrm{~km}$ squares. (D) we performed our analysis on four different spatial scales, while their analysis was performed on the single scale of $7.65 \mathrm{~km}$. (E) We concentrated on latent variables of pattern configuration while they look for latent variables of pattern structure including configuration and composition.

Our analysis has an advantage of being done on a much larger sample of landscapes coming from a single source, covering the entire world, and by considering patterns on multiple scales. However, because our landscape samples come in different sizes and shapes we used a smaller number of metrics in our analysis. Despite differences in the two methodologies, we find a correspondence between the results of the two analyses. Of the seven latent variables of landscape structure found by Cushman et al. four ("contagion/diversity", "large patch dominance", "interspersion/juxtaposition", and "patch shape variability") could be compared to our findings. The remaining three variables rely on metrics that we did not utilize for reasons stated above and thus cannot be compared to our findings. Comparing Table 6 in Cushman et al. paper with our Fig. 4 it follows that our complexity variable $C$ incorporates theirs "contagion/diversity" and "large patch dominance" variables, and our aggregation variable $A$ incorporates their "interspersion/juxtaposition", and "patch shape variability" variables. Thus, our findings agree broadly with Cushman et al. results and indicate that they could be valid over the entire terrestrial landmass and on multiple scales.

In addition to being a fundamental finding about spatial patterns of global land cover, an identification of complexity and aggregation as the two dominant descriptors of LP configuration greatly simplifies designing a classification of full landscape structures. In general, identifying a meaningful set of LPTs on the global scale is a daunting task due to a great diversity of observed structures. This is why a clustering approach to a delineation of global LPTs has limitations. A better strategy is to first disentangle an information about pattern configuration from the full pattern structure and then subdividing each configuration class into subclasses based on the composition. Here we have shown that the first step of such strategy yields a very favorable result because variety of pattern configurations can be captured by only two variables making classification of a pattern on the basis of configuration relatively simple. The second step of the strategy requires additional research, beyond the scope of this paper.

A classification of land units into pattern configuration types has its own merit. A resultant map is a never before seen abstraction of landscape structures and provides insight into broad scale organization of terrestrial LPs. It reveals that $56 \%$ of the landmass is covered by patterns having low complexity $(C=$ simple $)$. Examples of such landscapes 
include forest, bare areas, sparse vegetation areas, snow/ice areas, shrublands, and grasslands - all characterized by large expanses of mostly homogeneous land cover. Moreover, 39\% of the landmass is covered by simple, aggregated patterns - vast expanses of highly homogeneous land cover. On the other hand, only $4.8 \%$ of the landmass is covered by complex patterns and only $0.1 \%$ of the landmass is covered by complex, disaggregated patterns. This leaves about $40 \%$ of landmass covered by intermediate complexity patterns, $85 \%$ of which are also patterns of intermediate or high aggregation - expanses of land with the prevalence of one or two land cover classes. The map of LP configuration types shows that landscapes on Earth are mostly segregated by the land cover type. Complex landscapes with a diverse mix of different land cover types are rare exceptions from the prevailing monothematic cover. Future work will attempt to find major factors responsible for such organization of terrestrial landmass.

Acknowledgments. This work was supported by the University of Cincinnati Space Exploration Institute and by the grant NNX15AJ47G from the National Aeronautics and Space Administration.

\section{References}

Adams, R., Bischof, L., 1994. Seeded region growing. IEEE Transactions on Pattern Analysis and Machine Intelligence 16 (6), $641-647$.

Bailey, R. G., 1989. Explanatory supplement to Ecoregions Map of the Continents. Environmental Conservation 16(4), $3007-309$.

Barnsley, M., Barr, S., 1996. Inferring urban land use from satellite sensor images using kernel-based spatial reclassification. Photogrammetric engineering and remote sensing $62(8), 949-958$.

Bartholome, E., Belward, A. S., 2005. GLC2000: a new approach to global land cover mapping from Earth observation data. International Journal of Remote Sensing 26(9), 1959-1977.

Cardille, J. A., Lambois, M., 2009. From the redwood forest to the Gulf Stream waters: human signature nearly ubiquitous in representative US landscapes. Frontiers in Ecology and the Environment 8(3), 130-134.

Chang, P., Krumm, J., 1999. Object recognition with color cooccurrence histograms. In: In Proceedings of IEEE Conference on Computer Vision and Pattern Recognition, Fort Collins, CO, June 23-25, 1999. IEEE Computer Society Conference.

Chen, J., Chen, J., Liao, A., Cao, X., Chen, L., Chen, X., He, G., Lu, M., Zhang, W., Tong, X., Millis, J., 2014. Global land cover mapping at 30m resolution: A POK-based operational approach. ISPRS Journal of Photogrammetry and Remote Sensing.

Cushman, S. A., McGarigal, K., Neel, M. C., 2008. Parsimony in landscape metrics: strength, universality, and consistency. Ecological indicators $8(5), 691-703$

Eyre, M., Rushton, S., Luff, M., Telfer, M., 2004. Predicting the distribution of ground beetle species (Coleoptera, Carabidea) in Britain using land cover variables. Journal of Environmental Management 72, 163-174.

Fahrig, L., 2003. Effects of habitat fragmentation on biodiversity. Annual review of ecology, evolution, and systematics 34(1), 487-515.

Fuller, R. M., Devereux, B. J., Gillings, S., Amable, G. S., Hill, R. A., 2005. Indices of bird-habitat preference from field surveys of birds and remote sensing of land cover: a study of south-eastern England with wider implications for conservation and biodiversity assessment. Global Ecology and Biogeography 14, 223-239.

GRASS Development Team, 2016. Geographic Resources Analysis Support System (GRASS) Software. Open Source Geospatial Foundation, USA.

URL http://grass.osgeo.org

Haines-Young, R., Chopping, M., 1996. Quantifying landscape structure: a review of landscape indices and their application to forested landscapes. Progress in Physical Geography 20(4), 418-445.

Haralick, R. M., Shapiro, L. G., 1985. Image segmentation techniques. Computer vision, graphics, and image processing 29(1), pp.100-132. 29(1), $100-132$.

Heikkinen, R. K., Luoto, M., Virkkala, R., Rainio, K., 2004. Effects of habitat cover, landscape structure and spatial variables on the abundance of birds in an agricultural-forest mosaic. Journal of Applied Ecology 41, 824-835.

Homer, C., Dewitz, J., Yang, L., Jin, S., Danielson, P., Xian, G., Coulston, J., Herold, N., Wickham, J., Megown, K. C., 2015. Completion of the 2011 National Land Cover Database for the conterminous United Statesrepresenting a decade of land cover change information. Photogrammetric Engineering \& Remote Sensing 81(5), 345-354.

Jasiewicz, J., Netzel, P., Stepinski, T., 2015. GeoPAT: A toolbox for pattern-based information retrieval from large geospatial databases. Computers \& Geosciences 80, 62-73.

Jasiewicz, J., Stepinski, T. F., Niesterowicz, J., 2017. Multi-scale segmentation algorithm for pattern-based partitioning of large categorical rasters. Computers \& Geosciences submitted.

Kaiser, H. F., 1958. The varimax criterion for analytic rotation in factor analysis. Psychometrika 23(3), 187-200.

Kupfer, J. A., Gao, P., Guo, D., 2012. Regionalization of forest pattern metrics for the continental United States using contiguity constrained clustering and partitioning. Ecological Informatics 9, 11-18.

Lin, J., 1991. Divergence measures based on the Shannon entropy. IEEE Transactions on Information Theory 31(1), 145-151.

Long, J., Nelson, T., Wulder, M., 2010. Regionalization of landscape pattern indices using multivariate cluster analysis. Environmental Management 46(1), 134-142.

Luoto, M., Heikkinen, R. K., Pöyry, J., Saarinen, K., 2006. Determinants of biogeographical distribution of butterflies in boreal regions. Journal of Biogeography 33, 1764-1778.

McGarigal, K., Cushman, S. A., Neel, M. C., Ene, E., 2002. FRAGSTATS: Spatial Pattern Analysis Program for Categorical Maps (Version 3.3). Tech. rep., The University of Massachusetts: Amherst, MA, USA 
Niesterowicz, J., Stepinski, T. F., 2013. Regionalization of multi-categorical landscapes using machine vision methods. Applied Geography 45 (2013): 250-258. 45, 250-258

Niesterowicz, J., Stepinski, T. F., 2016. On using landscape metrics for landscape similarity search. Ecological Indicators 64, 20-30.

Niesterowicz, J., Stepinski, T. F., 2017. Pattern-based, multi-scale segmentation and regionalization of EOSD land cover. Int. J. Appl. Earth Obs. Geoinf. 62, 192-200.

Niesterowicz, J., Stepinski, T. F., Jasiewicz, J., 2016. Unsupervised regionalization of the conterminous U.S. into hierarchical landscape pattern types. International Journal of Geographical Information Science 30(7), 1450-1468.

Olson, D. M., Dinerstein, E., d. Wikramanayake, E., Burgess, N. D., Powell, G. V., Underwood, E. C., D'amico, J. A., Itoua, I., Strand, H. E., Morrison, J. C., j. Loucks, C., 2001. Terrestrial Ecoregions of the World: A New Map of Life on Earth A new global map of terrestrial ecoregions provides an innovative tool for conserving biodiversity. BioScience 51(11), 933-938.

Omernik, J. M., Griffith, G. E., 2014. Ecoregions of the conterminous united states: evolution of a hierarchical spatial framework. Environmental management 54 (6), 1249-1266.

Partington, K., Cardille, J. A., 2013. Uncovering dominant land-cover patterns of Quebec: Representative landscapes, spatial clusters, and fences. Land 2(4), 756-773.

Pfeifer, M., Disney, M., Quaife, T., Marchant, R., 2012. Terrestrial ecosystems from space: a review of earth observation products for macroecology applications. Global Ecology and Biogeography 21(6), 603-624.

Riitters, K. H., 2011. Spatial patterns of land cover in the United States: a technical document supporting the Forest Service 2010 RPA Assessment. Tech. Rep. Gen. Tech. Rep. SRS-136, Department of Agriculture Forest Service, Southern Research Station, Asheville, NC.

Riitters, K. H., Wickham, J. D., Vogelmann, J. E., Jones, K. B., 2000. National land-cover pattern data. Ecological Archives E081-004-D1.

Siriwardena, G. M., Crick, H. Q. P., Baillie, S. R., Wilson, J. D., 2000. Agricultural land-use and the spatial distribution of granivorous lowland farmland birds. Ecography 23, 702-719.

Tsendbazar, N. E., DeBruin, S., Herold, M., 2015. Assessing global land cover reference datasets for different user communities. ISPRS Journal of Photogrammetry and Remote Sensing 103, 93-114.

Tuanmu, M. N., Jetz, W., 2014. A global 1-km consensus land-cover product for biodiversity and ecosystem modelling. Global Ecology and Biogeography 23(9), 1031-1045.

Wickham, J. D., Norton, D. J., 1994. Mapping and analyzing landscape patterns. Landscape Ecology 9(1), 7-23. 